\title{
5. DER TRIALOGUS CVP 2868 UND SEINE TEXTTRADITION
}

Auch in der lateinischen Selbstbiographie Kaiser Maximilians über seine Erziehung findet sich der Hinweis auf geheim verfolgte Interessen: Er sei von den Historien und Taten der großherzigen Könige und Fürsten, von dem Erlernen (moderner) Fremdsprachen und der Jagd begeistert gewesen. Heimlich habe er diese Dinge treiben müssen ... und sei deshalb in große Unlust und Traurigkeit verfallen ${ }^{151}$. Und er berichtet - parallel zum Weißkunig - von slawischen Sprachbemühungen in der frühen Kindheit und deren Erneuerung im Mannesalter: Studuit linguam Latinam et quasi summarium omnium arcium liberalium, didicit quoque artem Schlavonicam ab uno rustico faceto et duobus suis pueris nobilibus ex Schlavonia. Tamen a magistro suo fuit impeditus, quod perficere non potuit, tamen in adulta aetate restauravit ${ }^{152}$.

Der Zusammenhang dieser Aussagen mit dem Wiener CVP 2868, einer ehemaligen Ambraser Handschrift, wurde bereits früh erkannt ${ }^{153}$. Allerdings verwechselten - wie bereits erörtert - die Haushistoriker der Wiener Hof- bzw. Nationalbibliothek den CVP 2868 verschiedentlich wohl mit dem CVP 2945. Der ehemals tirolische Kodex ist, wie die Schlußschrift vermerkt, als ein Trialogus bonus ac utilis mit In Vigilia Nativitatis 1489 datiert (vgl. Abb. 40).

Das 47 Papierfolien umfassende und auch im Format $(299 \times 215 \mathrm{~mm})$ durchaus repräsentative „Luxuswörterbuch" ${ }^{\text {154 }}$ wurde in einer „Kanzleikalligraphie“155 mit rubrizierten Überschriften, Fleuronnéeinitialen und Zierstrichen geschrieben (vgl. auch Abb. 39). Der haustypische „Van Swieten-Einband“ aus Pergament mit zweifacher Doppeladlerprägung ist mit 1753 datiert. Hauptstück des Kodex ist ein systematischer lateinisch-deutsch-tschechischer Nomenklator, dessen alltagssprachliches Spektrum von Gott bis zu den Insekten reicht und in einem Anhang ab 36v eine alphabetisch geordnete Liste von Verben, meist infinitivisch oder in erster, seltener in dritter Person Präsens bringt. Die beiden Wasserzeichen - je eine Waage mit und ohne Stern - lassen sich nicht zuverlässig einordnen.

Die Handschrift ist durch einen Nässeeinbruch beschädigt, der vor 1753 entstanden ist, da Vorsatzblätter und Pergamentbindung davon nicht betroffen sind. Er hat die falznahen Folienteile so stark angegriffen, daß durchgehend Flecken entstanden und das Rot von Initialen sich in die gegenüberliegenden Seiten einprägte. Deutlich sichtbar ist der Feuchtigkeitsschaden in Abb. 40. Es wäre nicht auszuschließen, daß er bis auf das Jahr 1665 zurückgeht und mit der abenteuerlichen Überstellung der Ambraser Bibliothek in die Wiener Palatina zusammenhängt. Der damalige Hofbibliothekar Peter Lambeck berichtet in diesem Zusammenhang über passive Widerstände und den Transport in Saltz-pantzen (Salzfässern) und Kisten über Inn und Donau: ... perrexi Vindobonam non minus periculose quam incom-

\footnotetext{
151 Vgl. Fichtenau, Maximilian (zit. A. 146) 33.

152 F. Schмid, Eine neue Fassung der maximilianeischen Selbstbiographie. Diss Wien 1950, 6.

153 Vgl. GotTlieb, Büchersammlung (zit. A. 108) 18.

154 So der Ausstellungskatalog Der Aufstieg eines Kaisers: Maximilian I. von seiner Geburt bis zur Alleinherrschaft. Wiener Neustadt 2000, 179.

155 Charakterisierung Franz Unterkirchers im Katalog Ambraser Kunst- und Wunderkammer. Die Bibliothek. Wien 1965, 45 .
} 
mode. Navis enim, quam multis flagitationibus vix tandem extorseram, valde fuit lacera \& ruinosa ${ }^{156}$. Das Titelblatt des Trialogus zeigt übrigens die nach der Transaktion von Lambeck eigenhändig und energisch geschriebene alte Ambraser Signatur MS Ambras.49\% (Abb.39) für das Antiquum quoddam Lexicon Latino-Germanicum-Bohemicum chartaceum in folio, scriptum A(nno) $1489^{157}$.

An der Provenienz aus Kaiser Maximilians Bibliothek kann schwerlich gezweifelt werden, findet sich unser Kodex doch im Inventari etlicher buecher so in ainem gewelb zu Ynnsprugk ligen für Erzherzog Ferdinand unter Nr.288. Ain Lateinischer teutscher vnd Behemischer vocabulari in rot leder gepunden von pogen plettern ${ }^{158}$. So gewiss nun aber die Abfassung des Verzeichnisses in die Zeit Ferdinands I. fällt, so sicher zeigt die Analyse seines Inhalts und die Identificierung der darin beschriebenen Handschriften und Drucke, dass wir es mit einer älteren, schon zu Maximilians I. Zeiten bestehenden Sammlung zu thun haben ${ }^{159}$. Damit erledigt sich auch eine vereinzelte Zuordnung des CVP 2868 zu jenen Handschriften, die der spätere Kaiser Ferdinand während seiner böhmischen Statthalterschaft erworben hatte ${ }^{160}$. Aufgrund der weihnachtlichen Datierung des Jahres 1489 könnte sogar vermutet werden, daß Maximilian den Trialogus in Linz übernommen hat, denn sein Itinerar hat einige Linzer Belege für November, und noch am 29. Dezember urkundet er in dieser Stadt ${ }^{161}$.

Die Verwandtschaft des Trialogus mit dem ältesten kompletten dreisprachigen Druck unter Einbeziehung der tschechischen Sprache überhaupt, dem Dictionarius: trium linguarum latine. teutonice: Boemice potiora vocabula continens: peregrinantibus apprime utilis, der 1513 in Wien erschien, hat erstmals Ferdinand Menčik erkannt ${ }^{162}$. Dieses Buch ist auch der früheste datierte dreisprachige Druck seiner Art, wenngleich ein 1968 in der Strahover Bibliothek gefundenes Fragment etwas vorangehen dürfte (Abb. 42). Unsere synoptische Edition bestätigt, daß es sich beim CVP 2868 und dem Trialogus um ein- und dieselbe Texttradition handelt. Der Druck von 1513 hat nur die Abfolge der Sachkapitel ab 18v blockweise zerrissen, was seine Abhängigkeit vom Strahover Fragment nahelegt, weil er damit den Formalfehler eines dortigen Bogendruckes übernimmt ${ }^{163}$. Weiters hat der Dictionarius statt der die Handschrift abschließenden alphabetischen Liste von Zeitwörtern einen Numerus von 1 bis 1000 angehängt. Die näheren Zusammenhänge werden im nächsten Kapitel erörtert.

Mit der vorliegenden Ausgabe wird ferner endgültig fixiert, daß der Claretus - und zwar das Glossar - nicht nur die Hauptquelle unserer Überlieferung ist, daß der Trialogus vielmehr vollständig von der älteren Überlieferung abhängt. Auf sie sind nämlich fast alle der 1884 lateinisch-tschechischen Wortgleichungen im handschriftlichen Nomenklator zurückzufüh-

156 P. Lambeck, Commentarii II. Wien 1669, 745. Eine Zusammenfassung der unerfreulichen Ereignisse findet sich bei H. Modern, Die Zimmern'schen Handschriften der K. k. Hofbibliothek. Ein Beitrag zur Geschichte der Ambraser Sammlung und der k. k. Hofbibliothek (JKhSW 20) Wien 1899, 1-2.

157 Ebenda, 988.

158 GotTlieb, Büchersammlung (zit. A. 138) 106. Das Bücherverzeichnis liegt als CVP $7999^{\circ}$ in der Handschriftensammlung der Österreichischen Nationalbibliothek.

159 Ebenda, 75 .

${ }_{160}$ Ambraser Kunst- und Wunderkammer (zit. A. 155) 42, ohne nähere Begründung.

${ }_{161}$ Freundliche Mitteilung Herrn Dr. Manfred HoldegGers von der Grazer Arbeitsstelle für die Regesten Maximilians I.

162 F. Menčík, Prešpurský slovník. Vocabularium latino-bohemicum Posoniense (Rozmanitosti. Př́spĕvky k dějinam starši české literatury 2). Prag 1892, XXXVIIIf.

${ }_{163} \mathrm{Vgl}$. dazu S. 64 f. 
ren. Ausnahmen sind Aurifodina|Zlata Jamma (3r, Z. 19), vernisur(a)/Vernis (6 ${ }^{r}, Z$ Z. 17), Liniale/Lyni (6r, Z. 26), Sopitus/Wspen (7', Z. 2), Strenuus/Bistry (7v, Z. 3), Decepcio/Pryelczeny (8', Z. 9), Armelezia/Wappn(n)rok (10', Z. 23), Mutta/Met (13', Z. 5), Puericia/Dytistwy (18v8), Strangwinea/Szabca (19v , Z. 28), Mortarium/Kubanie (22 $\left.{ }^{\mathrm{r}}, \mathrm{Z} .2\right)$, Rinensis/Reynsky (25), Z. 24), Sensualitas/Czilost (25, Z. 4), Reprehensio/Wynuge (27', Z. 14), allenfalls Ligacio/ Zatrzwenye (28v , Z. 14) und carinus/cernica $\left(36^{\mathrm{r}}, \mathrm{Z} .10\right)$. Dazu kommen, nur im Wiener Druck, Essiuride/grinsspat (nach 3v , Z. 18), Lapsata/sslaferna bzw. Scabor/wosnay nuž (nach 10 Z. 14), Scoreator/koniedra (nach 10', Z. 18), Triton/ktoznitzneumidielati (nach 18, Z. 4), musio/ morska kocžka (nach 18, Z. 15), Altum/hlubina (nach 31', Z. 8), Rubecula/ryba tzerwena (nach $32^{\mathrm{v}}$, Z. 4) susculus/prafatko (!) (nach 34 ${ }^{\mathrm{r}}, \mathrm{Z} .24$ ), rascula/wssy wlunie (nach $35^{\mathrm{v}}$, Z. 3) und cumes/hustze (nach $35 \mathrm{v}^{5}, \mathrm{Z}$. ).

Auf die enge Bindung Claretus-Trialogus hat zuerst Václav Flajšhans aufmerksam gemacht, der u.a. das Mißverständnis cochitus/horutzye im Trialogus (31', Z. 25) heranzog, das aus Kontamination der Klaretzeile 324 Cochitus plapolet, flegeton horuczen entstanden ist ${ }^{164}$.

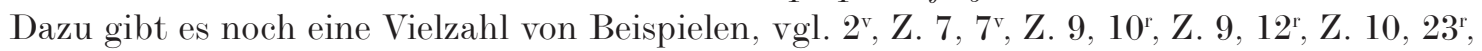
Z. $24 / 25,27^{v}, Z .15 / 16,32^{\text {r }}$ Z. 12 , aber auch Übernahmen von Irrtümern, vgl. etwa lateinisch Spinx, Albita (18 ${ }^{\mathrm{r}}, \mathrm{Z} .6$ und 9) oder Auisa $\left(21^{\mathrm{r}}\right.$, Z. 22).

Gelegentlich hat unser Text sogar fälschlich einen Numerus aus dem Klaretglossar übernommen, der dort aus Reimzwang bestand: Unsere Einzahlform Nakowonek zu Currigal $\left(10^{\mathrm{v}}\right.$, Z. 29) wird durch Klaret 2156 Collibar obogek, sido twrd, curigal nakolenek erklärbar, hrady/ castrum $\left(9^{r}\right.$, Z. 2) bezieht sich auf Klaret 2088 Vallus slup, vallum parkan, traco zkup, hradi castrum.

Dazu stellen sich offensichtliche grobe Abschreibfehler vom Typ Prumlna (27 $7^{\mathrm{r}}$ Z. 27, vgl. przymluva in Klaret 1432) oder Ysilobnik (30', Z. 20, vsilownik, Klaret 1233) usw. Die unserer Edition beigegebenen Versnummern des Klaretglossars belegen überdies, daß der Trialogus über weite Strecken auch der Binnengliederung der versifizierten Vorlage folgt, vgl. etwa die Kapitel De carmi (n)e et ip (s)ius attinencys $\left(6^{\text {r }}, Z\right.$. 11-6, Z. 1), de Imp (er)io et Regio ( $\left.n\right)$ ib (us) p(ro)ui(n)ciar(um) $\left(6^{\mathrm{r}}, \mathrm{Z}, 2\right.$ bis $7^{\mathrm{r}}, \mathrm{Z}$. 10) und de iudicio $\left(7^{\mathrm{r}}, \mathrm{Z} .11-8^{\mathrm{r}}, \mathrm{Z}, 28\right)$ mit Klaret De scriptoribus, De hominibus secularibus und De iudice. Der Trialogus beruht also auf einem Auszug aus jenem von Karl IV. veranlaßten Glossar in Versform, mit dem die systematische lateinisch-tschechische Lexikographie gleich am Beginn einen Höhepunkt fand.

Erst nachträglich wurde - zumindest teilweise - der deutsche Text ergänzt, sicher von einem Tschechen. Eine ganze Reihe deutscher Umsetzungen, vor allem Abstracta, sind nämlich offensichtlich Kunstwörter, die der lebenden Vulgärsprache fremd waren und mit ihren lateinisch-tschechischen Entsprechungen im späteren Druck, der Unverständliches ausmerzt, fehlen: Rector-Aufrichter (5 ${ }^{\mathrm{v}}, \mathrm{Z} .7$ ), Poeta-Mersager (5, Z. 3), Rhetoricus-Beschöner der $R^{\mathrm{e}} d$ (5v14), wahrscheinlich auch die wörtliche Übertragung Subplantator-Obstösser (5v, Z. 15), Loycus-Berediger (5v, Z. 19, falls es sich nicht von „Prediger" ableitet), Jurista-Puchrechter $\left(6^{\mathrm{r}}\right.$, Z. 4), Sophista-Listredner (6 ${ }^{r}$, Z. 5), Pegasus-Khunstperck ( 18 ${ }^{r}$, Z. 23), Aroma-Gutrauch (20', Z. 14), Continencia-widerhaldung (26r, Z. 3) usw. Andere sind im Druck korrigiert, vgl. etwa Quantitas-Wiegrossekait (2r, Z. 4) zu großheit, vielleicht auch Discipulus-Lerknecht (5v, Z. 12) zu iunger, Franngkreich(er) (25, Z. 26) zu frantzoß, Sensualitas-Mergklichkait (25v, Z. 4) zu synheit, Detractio-Affterlösung (27 ${ }^{\mathrm{r}}$, Z. 21) zu hynderrede usw. Presumcio-Getrawkeit(!)-Doufa-

164 Fla.šHans, Klaret (zit. A. 75) XXXI. Alle im Folgenden mit Klaret gekennzeichneten Zeilennummern beziehen sich auf diese Ausgabe. 
nie $\left(26^{\mathrm{v}}, \mathrm{Z} .13\right)$ kehrt $27^{\mathrm{r}}, \mathrm{Z} .13$ als Presumcio-darstocz mit der nun auch dem Sinn nach falschen und im Druck übernommenen Eindeutschung Getürstigkayt wieder. Dictator-Tichter (5 $\left.{ }^{\mathrm{v}}, \mathrm{Z} .10\right)$ steht in beiden Überlieferungen und ist dem Mittelhochdeutschen nicht fremd, vgl. allerdings die Übertragungen Dictamen-Getichte/Zlosek (7v, Z. 21, Druck vloženi), Poeta-werssow skladateltichter (Druck, nach 5 ${ }^{\mathrm{v}}$, Z. 10) gegen Poeta-Mersager-Skladatel (5 $\left.{ }^{\mathrm{v}}, \mathrm{Z} .3\right)$ und VersificatorVersmach(er)-Versobnik (5 ${ }^{v}, Z$. 11, fehlt im Druck), die semantische Unsicherheit signalisieren, vgl. auch Fabulor-Mersage-Bagy (41 ${ }^{\mathrm{r}}, \mathrm{Z}$. 2). Bemerkenswert ist in diesem Zusammenhang noch Qualitas-Wietennkhait (2' Z. 3) und seine druckerische Umsetzung mit vndertenigkeit, was sich am ehesten als Fehlinterpretation nach einer Vorlage Wietennkeit - wie im Kodex - erklären läßt ${ }^{165}$. Abschließend sei auf die holprige Umsetzung von ridiculosus-lachterig $\left(30^{v}, Z\right.$. 2) verwiesen.

Es gibt auch ungewöhnliche Verdeutschungen aus dem Tschechischen: SerophagiaNaworn-Nawora (21' ${ }^{\mathrm{r}}$ Z. 19, fehlt im Druck), Pluteum-Podgrad-Pohradka (22' , Z. 28, fehlt im Druck), wohl auch Cinamoniu(m)-Cymen-Czymny (20 ${ }^{\mathrm{r}}$ Z. 17, fehlt im Druck), BibulaPochnetz(!)-Petznetz (21 $\left.{ }^{\mathrm{r}}, \mathrm{Z} .24\right)$. Bemerkenswert ist ferner die gleichsinnige Übertragung von Gignosophista $\left(18^{\mathrm{r}}\right.$, Z. 19, fehlt im Druck) bzw. Antidotum $\left(20^{\mathrm{v}}\right.$, Z. 8) in beiden Vulgärsprachen: Arm (m)clug-Chudomuk (richtig Chudomudrak) bzw. Widertot-Protydan (20v, Z. 8) im Druck lekarzstwi-widerthan). Gelegentlich scheint sogar das lateinische Lemma vom Tschechischen beeinflußt: Tepa-Tepla woda (31 ${ }^{v}$, Z. 20, wohl statt Tepor, fehlt im Druck) oder Arx statt pinnaculum zu Arkyrz (22 $2^{v}$, Z. 5). Umgekehrt sind zumindest Impulsus/Ingetribnn ( $7^{\mathrm{r}}, \mathrm{Z} .26$, fehlt im Druck), Insanus/Vnsanig (20 r, Z. 3, fehlt im Druck) merkwürdige Latinismen im deutschen Text, weitere, anderswo nicht belegte deutsche Ausdrücke sind etwa Cometa/ Anczaichn(n) $\left(2^{v}, \text { Z. 1) }\right)^{166}$ oder Weinwaffn(n) (= Beinwaffen „Beinschienen“, 10v, Z. 29). Ein bereits von Joseph Dobrowsky gerügter tschechischer Germanismus ist Ver/Lenecz (2v Z Z. 14, im Druck podletij) ${ }^{167}$, dem im mittellateinischen Teil etwa Losunga neben vulgärsprachlichem Lösung/Pernie entspricht (9r, Z. 26, fehlt im Druck).

Auch die abschließenden Capitola de Verbis ab $36^{v}$, die im Druck fehlen, bestätigen mangelhafte Kenntnis der deutschen Sprache. Viele Zeitwörter in den Capitola De Verbis (36 ${ }^{\mathrm{vf}}$.) mit trennbarer Vorsilbe werden so flektiert, als seien Stamm und Präfix untrennbar: ich hingee, ich hinwirf, ich absneide, ich anneme, ich annhang, ich zulauff, ich vmbtrag, ich mitwon, fabulor/ich mersage (!), ich fridmach. Völlig ungewöhnlich sind auch einige verbale Umsetzungen aus dem Lateinischen, vgl. Aduersor/Widerwertig (36 ${ }^{\mathrm{v}}$, Z. 22), Adulor-Zuliebe (37 ${ }^{\mathrm{r}}, \mathrm{Z}$. 23), Auerto/Vonkere (38, Z. 8) usw. Vor allem deshalb vermutete schon Rudolf Wolkan einen ...Überarbeiter, welcher der deutschen Sprache wenig mächtig war... ${ }^{168}$. Darüberhinaus zeigen gerade die Capitola de Verbis, wie oberflächlich insgesamt gearbeitet wurde, so, wenn gele-

165 Die früheren zweisprachigen Drucke - vgl. dazu das nächste Kapitel - schreiben weitenigkayt usw., die Leipziger Ausgabe von 1514 korrigiert zu schicklikayt, vgl. B. WižD̆ÁLková, Nejstarší známý tištěný vokabulár̆. Miscelanea odděleni rukopisů a vzácných tisků 1/II (1971) 84.

166 Die deutsche Umsetzung ist aufgrund der besonders im 15. Jahrhundert verbreiteten Ansicht vom Kometen als Künder von - meist unheilvollen - Ereignissen plausibel. Vgl.dazu u. a. A. Lнотsкy und K. Ferrari d'occhieppo, Zwei Gutachten Georgs von Peuerbach über Kometen (1456 und 1457). MIÖG 68 (1960) 268. In der weit verbreiteten Schedelschen Weltchronik von 1493 sind Komet und Mond als Unheilkünder abgebildet, s. O. MaZaL, Die Sternenwelt des Mittelalters. Graz 1993, 150-151.

167 Joseph Dobrowsky, Geschichte der Böhmischen Sprache und ältern Literatur. Ganz umgearb. Aufl. Prag 1818. 307.

168 Wolkan, Geschichte der deutschen Literatur (zit. A. 91) 64. 
gentlich vulgärsprachliche Umsetzungen konjugationsmäßig nicht dem lateinischen Muster entsprechen, vgl. etwa Abnego/Lewgnen $\left(36^{v}\right.$, Z. 9), Probo/Doliczuge $\left(45^{\mathrm{r}}, \mathrm{Z} .17\right)$ usw.

Natürlich ist anzunehmen, daß der deutsche Text noch in Böhmen ergänzt worden ist. Das könnte erklären, warum gelegentlich das mitteldeutsche Präfix vor-durchscheint, vgl. Interdictum-Vorbotnn-Zapowied (7 ${ }^{\mathrm{v}}$, Z. 22, im Druck vorbittung), dazu auch Vorbuget (!) (7v, Z. 9, verbunden), Vorgebung $\left(28^{\mathrm{r}}, \mathrm{Z} .2\right)$ oder daß vereinzelt typisch ostmitteldeutsch. $f$ für nhd. pf steht, vgl. Vexillum/Vanne (3v, Z. 26, fann). Im Druck steht sogar tschechisch flastr für deutsch pflaster 20, Z. 13). Darüberhinaus existieren einige unverschobene $p$-Formen, etwa Prunus-Plawmen (15, Z. 12, pflaumbaum) oder Ancus-Krappn(n) (10v, Z. 28, fehlt im Druck), wo tschechisch aber Kraff (!) steht, vgl. auch Cassida-Slappe-Slapp (10v, Z. 1, fehlt im Druck), Widhop (33v, Z. 10, im Druck widhopf).

Eine Erinnerung an Böhmen ist auch zweifellos der Umstand, daß der Trialogus - wie seine klaretanische Vorlage, aber in Gegensatz zu anderen vergleichbaren Glossaren - ein eigenes Kapitel über das Baden hat (31 $1^{v}, Z$. 4ff). Hier drängt sich der Gedanke an die balneologische Tradition Böhmens ${ }^{169}$ und ein damit wohl zusammenhängendes Phänomen auf: Nirgendwo sonst hat die Idee des Badens den Hof und die höfische Kunst sosehr geprägt wie im spätmittelalterlichen Prag. Hier sei nur an den „Orden vom Bad“ am dortigen Hof und auf das Motiv des Königs mit der Bademagd in den illuminierten Handschriften Wenzels IV. verwiesen, dessen Popularität sich noch in der Böhmischen Chronik Wenzel Hajeks von Libotschan spiegelt ${ }^{170}$. Sprachlich bemerkenswert in diesem Konnex sind zwei Wortgleichungen des Trialogus: die oben schon besprochene lateinische Form Tepa nach tschechisch Tepla woda (31 $\left.{ }^{\mathrm{v}}, \mathrm{Z} .20\right)$ und die tschechische Umsetzung von Balneo/Ich Bad mit Blaznize (38, Z. 12). Hiebei könnte man Verbalhornung von tschechisch v lázni sem „ich bin im Bad“annehmen, es wäre aber - im Sinn ambivalenter Beurteilung des mittelalterlichen Badevergnügens - doch auch ein Konnex zu blázniti, blázni(wati) se „ausschweifen, ein Narr werden“ denkbar ${ }^{171}$. Direkt auf Prag, dessen Wenzelsplatz zumindest mittelbar bis in die Zeit Karls IV. zurückgeht, könnte sich die Gleichung Platea/Gasse/Vlicze (9 ${ }^{\mathrm{r}}, \mathrm{Z} .17$, ähnlich auch im Druck) beziehen. Die zu erwartende (ober)deutsche Umsetzung wäre Platz. Noch das dreisprachige Wörterbuch des Pragers Caspar Zacharias Wussin von 1742 hat für platea ausschließlich ein gaß oder straaß, vlice ${ }^{172}$. Eine interessante Frage wäre auch, wieweit es Korrelationen zwischen dem Kapitel De Monstris (18', Z. 3-25) bzw. seiner klaretanischen Vorlage und den phantastischen figuralen Randleistenverzierungen der böhmischen Handschriften vor und nach 1400 geben könnte.

Sosehr der deutsche Text des Trialogus in einer Restschicht seine nördlichere Herkunft durchscheinen läßt, ist er in seiner Gestalt von 1489 nach Schreibform und Wortwahl klar bairisch-österreichisch. Dabei ist weiters erkennbar, daß der spätere Wiener Druck grobe Dialektismen zu vermeiden sucht. Nur der Trialogus hat etwa die Kennformen Ergetag neben dinstag, Pfincztag neben donrstag (4v , Z. 3 bzw.5), Beichbischolff (5 $5^{\mathrm{r}}, \mathrm{Z}$. 8, Druck weichbischof),

\footnotetext{
169 Einer der ältesten Brünner Drucke ist die Abhandlung eines Clement von Grätz, Von den heissen Bädern, 1495 .

170 Darüber ausführlich J. KRÁsA, Die Handschriften König Wenzels IV. Wien 1971, 65-113.

171 J. Jungmanx, Slownjk česko-némecký I. Prag 1835, 135. Vgl. auch F. MıkLosich, Etymologisches Wörterbuch dert slavischen Sprachen. Wien 1886, 13 decipere, scandalum praebere.

172 C. Z. Wussis, Lexicon tripartitum oder Teutsch-Lateinisch und Böhmisches Wörter-Buch .... Prag ${ }^{3} 1742$, 488 .
} 
knoflich (13', Z. 27, knoblach), Kerspawm (14', Z. 25, kirschbaum), Phersich 15', Z. 4 pfirsenbaum), Zannd, Zanndflewsch (16 ${ }^{\mathrm{v}}, \mathrm{Z} .17 / 18$, zan, zanfleisch), Tennkhannt (17 , Z. 4, linckehant), Schef, Grosschef (31 ${ }^{\mathrm{v}}$, Z. 23, 32 $2^{\mathrm{r}}$ Z. 1. 12, schiff, grosschiff), Tahen $\left(33^{\mathrm{v}}\right.$, Z. 9, tole), dazu - nur im Kodex auch begrifflich belegt - Schefman $\left(32^{\mathrm{r}}\right.$, Z. 5) die sagenhafte Figur der Berchta $\left(18^{\mathrm{r}}\right.$, Z. 7) oder das Deminutivum Kindlpet $\left(19^{r}, Z\right.$ Z 19). Erwartungsgemäß haben indes beide Überlieferungen das aus dem Tschechischen übernommene bairisch-österreichische Schibboleth Ewncze $\left(11^{v}, \text { Z. 20, Encze }\right)^{173}$. Auf die signifikanten b-w-Vertauschungen wird in diesem Kapitel etwas später noch kurz eingegangen.

Die Art der Überlieferung macht von vornherein wahrscheinlich, daß der Kompilator der zugrundeliegenden Vokabularfassung und der Schreiber des Trialogus nicht identisch sein können und tatsächlich trägt unsere Handschrift eindeutig kopialen Charakter. Schon die stattliche Reihe rein formaler Fehler läßt einen nicht sehr versierten Abschreiber erkennen. 4v, Z. 25 wurde offenbar auf eine aus dem Druck zu erschließende Folgezeile Episcopus mit den deutschen bzw. tschechischen Gegenstücken vergessen bzw. Archiepiscopus mit BischoffBiskup kontaminiert. Dafür taucht sechs Zeilen später tschechisch Arczibiskup in der Gleichung Archidiaconus-Ertzbriebster(!) auf. 2 $2^{\text {r Z }} 11$ ist - statt des nach Mors in der vorhergehenden Zeile zu erwartenden Vita - irrtümlich die auf 23v Z. 8 im richtigen Zusammenhang stehende Zeile Vas-Vas-Sud (Zud) eingesetzt ${ }^{174}$. Ähnliche Zeilendopplungen - nur in der Handschrift - finden sich 7v, Z. 10 usw. Bei den Jahreszeiten wird 2v, Z. 15 für Autumnus fälschlich Hromnicz geschrieben, wo der Druck richtig $p<0>d z y m i j$ setzt, wenig später $\left(4^{\mathrm{v}}\right.$, Z. 18) steht richtig Purificacio-Liechtmess-Hromnicze. Natürlich ergeben sich auch Mißverständnisse durch falsche Übernahmen aus Nachbarzeilen. Eindrucksvollstes Beispiel ist das Paar $36^{v}$, Z. 6/7 mit einer zusätzlichen falschen $n$ - $u$-Vertauschung:

wird gefolgt von

$$
\text { Eycio/Auffwirff/Zpern (!) }
$$

Abluo/Abwasche/Zperu.

Mehrere Male bereitet die Ähnlichkeit lateinischer Nomina Schwierigkeiten: 5 ${ }^{\mathrm{r}}$, Z. 19 bzw. 22 - beide Zeilen fehlen im Druck - verführte die optische Nähe von Conuentus und Conuentor dazu, daß der Schreiber in beiden Fällen Nagenigk bzw. Nagenik einsetzte, während Klaret zwischen ssnatek (1025) und nagimak (1026) unterscheidet. 5v, Z. 20 wird Computista mit Componista verwechselt, vgl. die Kette Componista-Gesanngsecz(er)-Poczetnik. Der Druck bringt hier richtig Computista-početnik-rechner, Klaret 1080 hat compotista-poczetnik. Ein ähnlicher Irrtum passiert auf 8r, Z. 15 Error-blud-irrung, wo unser Schreiber als deutsche Entsprechung Kriegsal einsetzt, also Error mit Terror gleichgesetzt hat. Bemerkenswert ist schließlich 2v, Z. 9, wo Pruina irrtümlich zu ruina verkürzt wurde. Damit ging der semantische Konnex mit der deutschen Entsprechung Reiff verloren, die mhd. ja auch „Strick, Seil“ bedeutet. Prompt wurde sie zu tschech. Prowacz(e), was vermutlich der Schreiber zu Piramarze machte. Hefmut(er) (19", Z. 1, „Hebamme“) kehrt versehentlich auf 19v Z. 18 wieder und fehlt im Druck: Colica-Hefmueter-Tatrzeny. Tschechisch Barbirz, vielleicht zu einer ver-

${ }^{173} \mathrm{Zu}$ den hier genannten Beispielen s. E. Kranzmayer, Die bairischen Kennwörter und ihre Geschichte. Wien 1960, passim.

174 Dieser Fehler wurde in das zweisprachige Vokabular von 1505 und die Leipziger Ausgabe von 1514 übernommen, vgl. Wiž̄̌álková, Vokabulář (zit. A. 165) 22, 54 und 100-101. 
lorenen Zeile „Haarschneider“ gehörig, ist ein Irrläufer in der Gleichung Fullo-Mentler $\left(9^{v}\right.$, Z. 25, fehlt im Druck). Auf 29v Z. 10 Profugus-Flieher ist ihm mit Luspesnik(!) das tschechische Pendant einer vermutlich vergessenen Anschlußzeile Despoliator-Räuber in die Feder gerutscht (fehlt im Druck, vgl. alttschech. lúpežnik). Die im Druck eliminierten Zeilen 31 Z. 25 Cochitus/Flannk(!)|Horntzye und 32r, Z. 20 Genimina/Prut/Kzentze schließlich sind irrtümlich in das Kapitel über das Wasser bzw. über die Fische gelangt.

Die reinen Abschreibfehler sind so zahlreich und auf fast jeder Seite ablesbar, daß hier eine Auswahl genügt, denn schon die Überschriften belegen des Schreibers bescheidene Lateinkenntnisse: De ventibus (2r ${ }^{\mathrm{r}}$, nach 12, De aere im Druck), De de Arboribus (14'v nach12, fehlt im Druck). Schnitzer im Kontext sind etwa Tonitruus (2 ${ }^{\mathrm{r}}$, Z. 19, Druck Tonitrus), Jeurnium (4 ${ }^{\mathrm{r}}, \mathrm{Z} .12$, Druck Jeiunium), dis $\left(4^{\mathrm{v}}, \mathrm{Z}\right.$. 1, statt dies), Astrutus (7v , Z. 9, Druck Astrictus), Valla $\left(9^{\mathrm{r}}\right.$, Z. 11, Druck Ualua), Lambarda (11 ${ }^{\mathrm{r}}$, Z. 7, Druck Bombarda), Zerra (10 ${ }^{\mathrm{r}}$, Z. 16, wohl mlat. Ferra, fehlt im Druck), Susellium (12 ${ }^{\mathrm{r}}$, Z. 1, Druck Subsellium), Oper (12 $2^{\mathrm{r}}$, Z. 7, Druck Opex), Crepido (14', Z. 3, statt Trepido, fehlt im Druck), Spinx (18, Z. 6, fehlt im Druck), Hagale (22v, Z. 7, fehlt im Druck, statt Magale), Andarium (22v, Z. 20, fehlt im Druck, statt Aridarium), wohl auch Cirocillus (24', Z. 2, Girgillus), Nausca (29', Z. 14, Nausea), Saltatator (29r, Z. 24, fehlt im Druck), Fumus (30', Z. 1, Funus), Egrius (30', Z. 15, Egregius), Litex (31' Z. 24, fehlt im Druck, recte latex), Conapulame(n) (31 ${ }^{\mathrm{v}}$, Z. 13, fehlt im Druck, wohl mlat. Convapulamen), Capico (32v , Z. 7, fehlt im Druck, mlat. Capica), Paledens (34', Z. 22, Palledrus), Exoluo (40v, Z. 4, recte exsoluo), Iterrogo-Frag (42 ${ }^{\mathrm{r}}$, Z. 18), Mechor (43', Z. 11, statt Mechar), Reccio (45, Z. 14, statt Recido) usw.

In gleicher Weise stand er mit dem Deutschen auf Kriegsfuß. In einigen Fällen, wo die Vorlage nicht entziffert werden konnte, entstanden besonders sinnstörende Buchstabenfolgen, die vom Druck bezeichnenderweise meist nicht übernommen oder korrigiert worden sind, vgl. etwa Nymbus-Sayffe(n) Regn (n) (2r, Z. 16, statt sanfft regen, vgl. im Druck nach $2^{r}$, Z. 17), enigma-retels $\left(6^{r}, Z\right.$ Z. 2 , im Druck nicht vorhanden), das schon erwähnte und dem Druck fehlende Ertzbriebster (5 ${ }^{\mathrm{r}}, \mathrm{Z} .5$ ), Conuentor-Mitherer-Nagenik (5r, Z. 22, wohl statt mietener, fehlt im Druck), Studium-Vlers (5 ${ }^{\mathrm{v}}$, Z. 27, wohl statt Lere, der Druck schreibt lernuug!), Laub 7v, Z. 6, Druck vrlaub), Exhibicio-Erpietung-Prczykazeny (8 ${ }^{\text {r }, ~ Z . ~ 12, ~ s t a t t ~ E r-~}$ preitung, fehlt im Druck), Theolonarius-Zoldner-Mytny (8v' Z. 6, czollner), Expedicio-Herschafft $\left(9^{\mathrm{v}}\right.$, Z. 4, herfart), Follis-Blospald-Myech $\left(10^{\mathrm{r}}, \mathrm{Z} .12\right.$, im Druck ploßback!), Khombt statt Khomat „Kummet" (11 ${ }^{\mathrm{v}}$, Z. 12, fehlt im Druck), Cornus-Puchen (15, Z. 7 statt Putten, im Druck haenbutte(n)baum), Oberbrach (16 ${ }^{v}$, Z. 4, statt Augenbra, die Fehlschreibung könnte vom tschechischen Oboczie beinflußt sein, im Druck augenprag), Languor-Traurigkhait (19r, Z. 21, Druck Krankheit), Kram (m) (19v, Z. 6, Druck krampff, vgl. tschechisch chrmtna), Cremiu (m)Grife $\left(21^{\mathrm{r}}\right.$, Z. 23, Druck Grube), Plenitudo-Volkhait $\left(26^{\mathrm{v}}\right.$, Z. 2, volheyt), Periculum-Verderbe-Pakost (28, Z. 17, fehlt im Druck), Torrens-Prud-Waldwasser (31 ${ }^{\mathrm{r}}$, Z. 3, statt Wildwasser, im Druck schnelwasser), Flannk statt Flamm (31'r, Z. 25, fehlt im Druck), AbortiuusVerwaffner-Zpratek (34 ${ }^{\mathrm{v}}$, Z. 23, statt Verworfner fehlt im Druck), Damma/Amster (35", Z. 2, hambster), Enodo-Aufkhümpf (40', Z. 20, statt Aufkhnüpf), Opilo-Ich v(er)stop (44r, Z. 7, statt Ich versto $\beta$ ). Falsche Übersetzungen sind vermutlich Puchstab für Gramaticus-Slowoczan (5 ${ }^{v}$, Z. 24, fehlt im Druck), zumindest eigenwillig Malignor-Zlostym sye als Frismich (43r3), Manduco-Gym als Ich Iss (43', Z. 7), wohl auch Erfare für Suscitor(!)-Spitawamsie (46 ${ }^{\mathrm{r}}$, Z. 6) u. a.

Die mindere Qualität des tschechischen Textes ist ein Hauptgrund dafür, daß sich auch die Bohemistik lange Zeit wenig um den Trialogus kümmerte. Schon Joseph Dobrowsky hatte den Vorwurf erhoben ...das Böhmische ist sehr fehlerhaft geschrieben ... und zeigte sich 
über die schlechte Abschrift geradezu verärgert ${ }^{175}$. Erst Ferdinand Menčík, der Slawist der Wiener Hofbibliothek dachte nach 1880 ernsthaft an eine Edition, eine Forderung, die wenige Jahre später Rudolf Wolkan aus germanistischer Sicht neu stellte ${ }^{176}$.

Hier seien zunächst einige Kopierfehler angeführt. Auf $4^{\mathrm{r}}$, Z. 27 steht fälschlich Modla für Oracio-Gepet, denn alttschech. Modla steht - etwa im Claretus 2334 - für ydola, „Götze“. Unser Schreiber hat offenbar eine Kontraktionskürzung der Vorlage übersehen, es müßte Modlitba stehen ${ }^{177}$. Der Druck schreibt movijtba. Ein weiterer verstümmelter Ausdruck der Sakralsphäre ist Zirbek (3v, Z. 22, statt Cierkev, fehlt im Druck). Problematisch ist die Zusammenstellung Registrum-Anricht(e)-Naprana (4 ${ }^{\mathrm{r}}, \mathrm{Z} .26$, Registrum-registra-register $)^{178}$. Vermutlich steht unser Text in Zusammenhang mit mhd. entrichten ,abgeben“, dem alttschechisch náprava „ersetzen“ entsprechen könnte. Naprana wäre somit ein Kopierfehler, Klaret 2380 schreibt für Registrum poprawna ${ }^{179}$. Weitere Unaufmerksamkeiten sind etwa Bezzny $\left(4^{\mathrm{v}}\right.$, Z. 20, statt Wzetye, fehlt im Druck), Przebnyl (4v, Z. 24, statt Przizednik, fehlt im Druck), Blenot (10v, Z. 24, statt Klenot, fehlt im Druck), Pastor-Patuscha (11 ${ }^{\mathrm{r}}$, Z. 21, Druck pastucha), Pohononicz (11 ${ }^{\mathrm{r}}$, Z. 23, Druck pohonicz), Weinper-Zrucze 14 ${ }^{\mathrm{r}}$, Z. 25, vgl. im Druck 14 ${ }^{\mathrm{v}}, \mathrm{Z} .1$ zrntze), Fagus-Kuk (15 $5^{\mathrm{v}}, \mathrm{Z} .2$, buk), quali $\left(21^{\mathrm{r}}\right.$, Z. 15, fehlt im Druck, vgl. alttschech. kvasy,), Ohorzalose (19v , Z. 27, statt Ohorzalost, fehlt im Druck), Santronk (27 ${ }^{\mathrm{v}}$, Z. 15, fehlt im Druck, alttschech. šandrok), Duo (30v , Z. 24, Dno), Situla-kber (31 ${ }^{v}$, Z. 21, fehlt im Druck, vgl. alttschech. kbel), Supyna (32r, Z. 21, vielleicht vom deutschen schuppe abgeleitet, im Druck lupina), Pobrha (32v, Z. 15, fehlt im Druck, vgl. alttschech. pobrza), Pluma-Oslnecz (fehlt im Druck, vgl. alttschech. ostnecz), Lewerburst-Giernitze $\left(34^{\mathrm{v}}, \mathrm{Z} .22\right.$, gitrnitze), cernica $\left(36^{\mathrm{r}}, \mathrm{Z} .10\right.$, wohl alttschech. ̌̌ervica).

Der Abschriftcharakter beweist sich übrigens auch für den zweiten alphabetischen Teil des Trialogus ab $36^{\mathrm{v}}$ in einer großen Zahl von kopialen Errata, vgl. etwa Ktwm statt kwu $\left(41^{\mathrm{r}}\right.$, Z. 11, vgl. 47 $7^{\mathrm{r}}$, Z. 8) oder Matzerugy statt Nacerugy $\left(41^{\mathrm{v}}\right.$, Z. 2) u.a. Bei Quantitas-Yakost $\left(2^{\mathrm{r}}\right.$, Z. 4, statt Kterakost) ist offensichtlich die Entsprechung für Qualitas aus der vorangehenden Zeile übernommen worden ${ }^{180}$. Auf $29^{v}$, Z. 15 Incantator-Zaubrer wurde alttschechisch zvábovnik mit zarodnik „Stammvater" verwechselt. Im Tschechischen sonst nicht belegt sind die vermutlichen Fehlschreibungen Przebnyl für Patriarcha (4 , Z. 24, Druck patriarcha) oder Pertink für Conuersus (5, Z. 17, fehlt im Druck). Dagegen bin ich mir nicht sicher, ob die Gleichung Ocras-

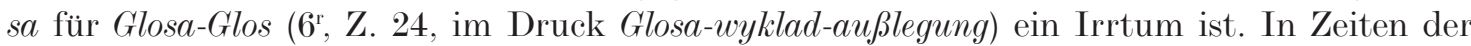
Scholastik und des „geblümten Stils“ ergäbe die durch Belege im Klaret und dem Staročeský šlovník abgesicherte Bedeutung „,rhetorische Figur, decorum“ durchaus einen Sinn.

Wo das tschechische dem deutschen Lemma entspricht, ist im Trialogus die tschechische (= letzte) Kolumne vielfach nicht ausgefüllt: 4 , Z. 23 Cardinalis/Cardinal, Papir(us)/Papir $\left(6^{\mathrm{r}}\right.$, Z. 27). Man vgl. ferner 20v , Z. 9, wo für Geripigra in der deutschen Kolumne Kruss steht, was genau alttschechischem hruš (Klaret 894 hruss) entspricht, 7, Z. 20 Priuilegium-hantuest, vgl. alttschech. hamferst (Klaret 2280), 23v, Z. 24 Modabrum-Streichholtz, vgl. alttschech.

175 Dobrowsky, Geschichte (zit. A. 177) 306-307. Vgl. auch FlajšHans, Klaret (zit. A. 75) XXXI.

176 Menčík, Prešpurský slovník (zit. A. 162) XXXII, bzw. R. Wolkan, Geschichte der deutschen Literatur (zit. A. 91) 64.

177 Übrigens schreibt auch der CVP 2945 falsch modlitha (335).

178 Zu Náprava vgl. V. BrandL, Glossarium illustrans bohemico-moravicae historiae fontes. Brünn 1876 , 169. Der Leipziger Druck von 1514 schreibt sogar Registrum/anrichter, vgl. WižĎÁLKovÁ, Vokabulár̆ (zit. A. 165), 84 .

179 Die Klaretnummern entsprechen generell der Ausgabe von V. FlajšHans, Klaret (zit. A. 75).

180 Vgl. dazu allgemein F. Oberpfalcer, Jakost. Naše řě X/7 (1926), besonders 193-194. 
strychholc (Klaret 2531), 32v , Z. 17 Ostrea-Snek, vgl. alttschechisch šnek, 32v , Z. 16 Strumul(us)Stogkhvisch, bei dem der Druck ein beweisendes tschechisches stokwiss ausweist. Einem typischen Kopistenfehler entspringt das Zeilenpaar 22r26 zu 22v' Z. 1:

\section{Fundamentu(m)-Gruntuest-Podlaha \\ Pauime(n)tum-Estreich-(kein Beleg).}

Fundamentum entspricht auch alttschech. krumfešt, die Stelle blieb ursprünglich leer und dem Schreiber ist hier irrtümlich die tschechische Gleichung für Pauimentum in die darüberliegende Zeile gerutscht. Bei $5^{v}, Z .18$ Documentum-lernu $(n) g$ dürfte das fehlende tschechische Lemma dem lateinischen Muster entsprechen, bei $5^{\mathrm{v}}$, Z. 15 Subplantator-obstösser läßt sich insgesamt keine parallele tschechische Entlehnung feststellen. Hier ist aber auch die ungebräuchliche deutsche Umsetzung einfach dem Lateinischen nachgebildet

Ferdinand Menčik vermutete aufgrund der Fehlschreibungen porneznik (5 $5^{\mathrm{r}}$ Z. 21), milostvroy $\left(4^{\mathrm{r}}, \mathrm{Z} .21\right)$ und sunabt $\left(1^{\mathrm{r}}, \mathrm{Z} .21\right)$, daß der Schreiber kein Tscheche gewesen sei ${ }^{181}$, und in der Tat sind grobe Mißverständnisse in dieser Sprache am häufigsten, wozu übrigens auch indistinkte Schreibungen der Art Prziezadastzep (14v, Z. 17) gehören. Erwähnt sei auch, daß die gelegentlichen bairisch-österreichischen b-w-Vertauschungen vom Typ Consolida/Weybel (13 ${ }^{v}$, Z. 24, im Druck beynwel) oder Lewerburst (34v , Z. 22, fehlt im Druck) auch im tschechischen Text begegnen, vgl. etwa Vertex/Burch 16r, Z. 23, fehlt im Druck) oder Dowybam (37", Z. 14, tschechisch dobývám). Man könnte aber aufgrund von nicht gerade seltenen Schnitzern wie Morch statt Morgen (12r, Z. 25), sneel statt snell $\left(30^{\mathrm{v}}\right.$, Z. 6), Holtzflos statt Holtzslos (23r, Z. 22) usw. auch deutsche Herkunft anzweifeln. Bedenkt man dazu die sinnstörenden Fehler im „neutralen“ Lateintext so bleibt die Feststellung, daß auch der CVP 2868 von einem unbedarften bzw. sorglosen Schreiber kopiert wurde, der seine Arbeit optisch befriedigend aber inhaltlich so flüchtig wie möglich erledigte. Von diesem Manko sind - nach dem Tschechischen - auch die beiden anderen Sprachen betroffen.

Vielleicht sollte das vorliegende Buch bzw. sein Besitz nur der Selbststilisierung eines polyglotten Herrschers dienen. Allein schon die geringe sprachliche Sorgfalt unserer Handschrift schließt aus, daß der Trialogus für Maximilian eigens zusammengestellt worden ist, auch wenn wir nicht wüßten, daß eine komprimierte Prosaauflösung des lateinisch-tschechischen Claretus zugrunde liegt. Besonders der sogenannte Klen Rozkochaný aus Raigern und die Preßburger Fassung beweisen, daß derartige Umsetzungen schon im vierzehnten Jahrhunderts beliebt waren. Auf keinen Fall war die Vorlage zum Trialogus ein lateinisch-deutsches Wörterbuch, dem dann die tschechischen Termini hinzugefügt wurden - sprachliche und überlieferungsgeschichtliche Gründe sprechen dagegen ${ }^{182}$. Die deutschen Übersetzungen dürften noch in Böhmen und wohl von einem Tschechen verfaßt worden sein.

Für den Regenten hat man dann einfach den Wortlaut der vermutlich auch in Österreich gängigsten Tradition abgeschrieben und eine Liste mit alphabetisierten Verben beigefügt. Damit erhielte das ...Trialogus bonus ac utilis... in der Schlußschrift einen einfachen Sinn. Man folgte dem Wunsch Maximilians nach einem „modernen“, praktischen Sprachbehelf, ähnlich dem Vokabular, das er in seiner Jugend gehabt hatte. Das Problem der Trialogusüberlieferung - im Kontext mit einschlägigen Drucken der ersten Hälfte des sechzehnten Jahrhunderts - wird gegen Ende des nächsten Kapitels nochmals zur Sprache kommen.

181 Menčík, Prešpurský slovník (zit. A. 162) XXVI. Vgl. dazu FlajšHans, Klaret (zit. A. 75), 80-89.

182 WiŽ̄̌ÁLKová, Vokabulář (zit. A. 165) 10, 20, 98-99, 158 und passim. 